ACCEPTED MANUSCRIPT

\title{
Design and testing of a microelectrode array with spatial resolution for detection of cancerous cells in mixed cultures
}

To cite this article before publication: Fabián Eduardo Giana et al 2019 Meas. Sci. Technol. in press https://doi.org/10.1088/1361-6501/ab4d35

\section{Manuscript version: Accepted Manuscript}

Accepted Manuscript is "the version of the article accepted for publication including all changes made as a result of the peer review process, and which may also include the addition to the article by IOP Publishing of a header, an article ID, a cover sheet and/or an 'Accepted

Manuscript' watermark, but excluding any other editing, typesetting or other changes made by IOP Publishing and/or its licensors"

This Accepted Manuscript is @ 2019 IOP Publishing Ltd.

During the embargo period (the 12 month period from the publication of the Version of Record of this article), the Accepted Manuscript is fully protected by copyright and cannot be reused or reposted elsewhere.

As the Version of Record of this article is going to be / has been published on a subscription basis, this Accepted Manuscript is available for reuse under a CC BY-NC-ND 3.0 licence after the 12 month embargo period.

After the embargo period, everyone is permitted to use copy and redistribute this article for non-commercial purposes only, provided that they adhere to all the terms of the licence https://creativecommons.org/licences/by-nc-nd/3.0

Although reasonable endeavours have been taken to obtain all necessary permissions from third parties to include their copyrighted content within this article, their full citation and copyright line may not be present in this Accepted Manuscript version. Before using any content from this article, please refer to the Version of Record on IOPscience once published for full citation and copyright details, as permissions will likely be required. All third party content is fully copyright protected, unless specifically stated otherwise in the figure caption in the Version of Record.

View the article online for updates and enhancements. 
Keywords: microelectrode array, spatial resolution, ECIS, cancer cells, mixed culture. Submitted to: Meas. Sci. Technol.

\begin{abstract}
We present the design, construction and testing of a microelectrode array with spatial resolution which can be used for the detection of cancerous cells in a mixed cell culture by means of the impedance spectroscopy technique. Two different microelectrode diameters, namely $57 \mu \mathrm{m}$ and $211 \mu \mathrm{m}$, were tested. A layer of an $\mathrm{AuCu}$ alloy was deposited between the glass substrate and the exterior Au layer, enhancing the adhesion of the microelectrodes to the substrate and at the same time showing lower impedance than the commonly used material Au. Characteristic features were extracted from the impedance measurements at different phases (growth, confluence, wound and healing) and used to feed a Linear Discriminant Analysis algorithm in order to discriminate between normal and cancerous cells. Relevant statistical tests were applied in the discrimination model for each phase. Finally, it was determined that the larger microelectrodes have a superior discriminant capacity: no incorrect classifications were obtained with microelectrodes measuring $211 \mu \mathrm{m}$ in diameter, while $23.5 \%$ false positives and $5.6 \%$ false negatives were obtained with $57 \mu \mathrm{m}$ microelectrodes.
\end{abstract}

\title{
Design and testing of a microelectrode array with spatial resolution for detection of cancerous cells in mixed cultures
}

\author{
F E Giana ${ }^{1,2,3}$, F J Bonetto B $^{1,2,3}$ and M I Bellotti ${ }^{1}$
}

${ }^{1}$ Laboratorio de Cavitación y Biotecnología, Centro Atómico Baríloche, San Carlos

2 Instituto Balseiro, Universidad Nacional de Cuyo - Comisión Nacional de Energía Atómica, San Carlos de Bariloche, R8402AGP, Argentina

${ }^{3}$ Consejo Nacional de Investigaciones Científicas y Técnicas (CONICET), Buenos

Aires, C1033AAJ, Argentina

E-mail: bellotti@cab.cnea.gov.ar, fabian.giana@ib.edu.ar

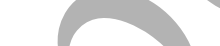

s.

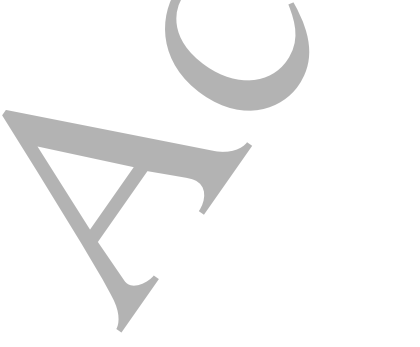




\section{Microelectrode array for detection of cancer cells}

\section{Introduction}

The Electric Cell-substrate Impedance Sensing (ECIS) technique is based on the culture of cells on a microelectrode array (MEA), which is a set of culture wells containing micrometer-sized electrodes, to study different properties of the cells by monitoring the changes in the system's electrical impedance. Particularly, this technique has been extensively employed in the field of cancer study [1] to investigate many aspects related to the behavior of cancer cells, such as carcinogenesis [2], cell adhesion, migration and proliferation $[3,4,5,6,7]$, cell-cell interactions [8] and tumor suppression [9, 10, 11].

In existing commercial ECIS devices [12], each well contains only one or two independent microelectrodes located approximately in the center of the circle that represents the culture area, and therefore don't allow for impedance spectral measurements with spatial resolution. There are also commercial arrangements of multiple electrodes connected in parallel, that is, they are not independent. In this case, the excitation signal affects all the electrodes simultaneously and the resulting measurement contains mixed information from different/regions of the culture (the effective area under analysis is the sum of the areas of all the microelectrodes involved). Moreover, inside a typical culture well the microelectrode normally covers only a very small portion of the total area, and therefore the information that can be obtained through impedance measurements is representative of an equally small portion of the cell layer. In many applications this is not relevant, since the system under study usually consists of a cell line, which presents homogeneous properties throughout the entire cell layer. However, this could be an issue within some frameworks, particularly when trying to achieve discrimination between normal and cancerous cells. In this context, a discrimination technique based on ECIS measurements was recently developed [13] using commercial MEAs and independent cultures of normal and cancerous cells. It is of our interest to apply this technique in a discrimination experiment where both normal and cancerous cells are mixed in a single culture.

The first difficulty that arises when designing an experiment of this kind lies in the fact that the electrical behavior of a culture with the mentioned properties is not well characterized. In a culture formed by identical cells, it is known that after inoculation the cells attach to the substrate and then migrate and spread over the available surface. Once the whole culture surface has been covered, the cells form tight junctions and the resulting stucture is known as a confluent monolayer (in the case of cancer cells, it is thought that they could form multilayers). Now, in the simplest case of a mixed culture, if a suspension containing exactly the same amount of normal and cancerous living cells is inoculated in the same well, it is expected that they will be initially distributed in a random fashion covering approximately equal areas on the culture and, once settled, begin to migrate and spread. However, in this case it is not clear if adjacent cells of different nature will form tight junctions and therefore a confluent monolayer. Even if a cell monolayer is formed in a mixed culture, it is expected that its morphological and structural characteristics will be heterogeneous and, consequently, so will their electrical 


\section{Microelectrode array for detection of cancer cells}

properties. This implies that impedance measurements carried out on different portions of the culture would yield different results. Therefore, using a single microelectrode with small surface area would not provide sufficient information to determine the properties of the mixed culture and, consequently, to detect the presence of cancer cells. In fact, the culture area in a typical commercial well [12] is approximately $32 \mathrm{~mm}^{2}$, while the area of the only microelectrode in the well is $0.05 \mathrm{~mm}^{2}$, which represents $0.16 \%$ of the total. Moreover, not only the total measurement area is important to detect the presence of cancer cells, but also the spatial distribution of the measurements throughout the entire culture area. Thus, in order to determine the presence of cancer cells in a heterogeneous culture as described above, it is necessary to obtain information from different parts of the cultured surface. In this work, we present the design of a microelectrode array which allows for the measurement of electrical impedance in different spatial regions of the same cell culture. With this design, the total measurement area increases up to 6 times in comparison with typical arrays and measurements can be carried out on 20 different parts of the culture. In addition, we tested two different microelectrodes diameters (57 and $211 \mu \mathrm{m}$ ) in order to determine the optimal conditions of the discrimination experiment.

\section{Materials and methods}

\subsection{Cell cultures}

In this work, all the experiments were carried out on two different cell lines, namely the normal line NMuMG (ATCC, CRL-1636), which comes from normal glandular tissue of mice [14] and the cancerous line LM3, which comes from murine mammary adenocarcinoma [15]. We used cell suspensions with passage numbers ranging from 8 to 15 and 6 to 12 for NMuMG and LM3 cells, respectively. Cells were cultured in an incubator at $37{ }^{\circ} \mathrm{C}$, humidified and containing $5 \% \mathrm{CO}_{2}$ [16]. The culture medium was the same for both cell lines and was composed of $57 \%$ Dulbecco's modified Eagle's medium (DMEM F-12, Gibco), 30\% modified Eagle's medium (MEM, Gibco), 10\% fetal bovine serum, $1 \%$ nonessential amino acids, and $2 \%$ HEPES buffer. The final pH of the medium was 7.4. Cell suspensions were prepared using standard trypsinization procedures $(0.05 \% \mathrm{w} / \mathrm{v}$ trypsin - $0.53 \mathrm{mM}$ EDTA $4 \mathrm{Na})$. Each electrode was seeded with $0.5 \mathrm{ml}$ of cell suspension at a density of approximately $1 \times 10^{6}$ cells.ml ${ }^{-1}$. The culture medium on the wells was changed every $12 \mathrm{~h}$, pausing the measurements for a total of $30 \mathrm{~min}$ to allow for temperature stabilization.

Before culturing the cells, the electrodes were sterilized in an oven at $180{ }^{\circ} \mathrm{C}$ for $2 \mathrm{~h}$ and then a pre-treatment step with a $10 \mathrm{mM}$ solution of L-cysteine in normal saline water was carried out in order to improve experiment repeatability [17]. In this treatment, $200 \mu l$ of the solution were added to each well and the system was left 15 min at room temperature before washing with deionized water. The electrodes were afterwards incubated with media for approximately $24 \mathrm{~h}$ before seeding the cells, to 


\section{Microelectrode array for detection of cancer cells}

allow proteins to pretreat the electrode surface, enhancing the attachment of cells.

\subsection{Measurement protocol}

Noninvasive impedance measurements were carried out by following a standard impedance spectroscopy scheme as described in a previous work [13], in which a function generator (Hewlett-Packard, HP33120A) applies a sinusoidal waveform of $100 \mathrm{~m} V_{R M S}$ to the active electrode through a $100 \mathrm{k} \Omega$ series resistor, producing noninvasive alternating currents with amplitudes less than $1 \mu \mathrm{A}_{R M S}$. A lock-in amplifier (Stanford Research Systems, SR530) measures the in-phase and out-of-phase voltages, quantities that are afterwards converted to real and imaginary parts of the system's impedance. The results are presented formally as resistance $(R)$ and capacitance $(C)$ of a series-equivalent $\mathrm{RC}$ circuit [18], which are computed from the real $(\Re)$ and imaginary $(\Im)$ parts of the complex impedance $Z$ for each frequency using the equations:

$$
\begin{aligned}
& R(f)=\Re\{Z(f)\} \\
& C(f)=-\frac{1}{2 . \pi . f . \Im\{Z(f)\}} .
\end{aligned}
$$

As can be seen, this simple model results in frequency-dependent parameters $(\mathrm{R}$ and $\mathrm{C}$ ), and it is used as an equivalent circuit representation in the framework of the ECIS technique. As was stated in a pioneering ECIS work, all other simple, equivalent representations of the interface also result in a frequency dependent resistance and capacitance [19].

We employed a measurement protocol developed in a previous work to discriminate between normal and cancerous cells based on impedance spectroscopy [13]. The measurements were carried out in four different consecutive phases as follows:

(i) Growth phase: the cells attach and spread over the entire culture surface, including the microelectrodes, and the system's impedance increases. In this phase, impedance was registered as a function of time at two different frequencies, namely $4775 \mathrm{~Hz}$ and $100 \mathrm{kHz}$, which were selected according to the sensitivity of the measurement [13]. Based on prior results, we designed this measurement to have a duration of $15 \mathrm{~h}$ in order to capture the whole dynamics of the process.

(ii) Confluence phase: after the cells have covered all the available surface, they form tight junctions and the resulting state is known as confluence. In this phase, we measured impedance at 6 logarithmically equally spaced frequencies ranging from $419 \mathrm{~Hz}$ to $29.6 \mathrm{kHz}$.

(iii) Wounding phase: during this phase, the confluent cell monolayer is deliberately damaged to carry out a wounding assay. A specific technique aimed to obtain information about the wounding dynamics has been recently developed [20] and used to discriminate between normal and cancerous cells [13]. We used a similar 


\section{Microelectrode array for detection of cancer cells}

technique in this work, with some variations. Measurements were carried out with an LCR-meter (Agilent Technologies, E4980a). The amplitudes of the wounding and control signals were $2 \mathrm{~V}_{R M S}$ and $20 \mathrm{mV}_{R M S}$, respectively, and the frequency was in both cases $30 \mathrm{kHz}$. The measurement cycle consisted of an alternation between high- and low-voltage measurements until reaching 60 seconds of exposure time to the wounding signal. Then, a high-frequency measurement at $2 \mathrm{~V}_{R M S}$ and 64 $\mathrm{kHz}$ was carried out for $60 \mathrm{~s}$ to ensure complete cell death over the mioroelectrodes before the healing phase [21].

(iv) Healing phase: finally, the noninvasive, lock-in based measurement scheme was used to register impedance as a function of time with the same amplitude and frequencies used in the growth phase, in order to monitor the dynamics of the healing process.

\subsection{Discrimination algorithm}

Following the method presented in a previous work [13], from the data obtained through the measurement protocol described in Section 2.2 we extracted characteristic features related to the specific behavior of both cell types. Each sample was associated with a vector $\mathbf{x}$, whose coordinates represent the values of each feature in a multidimensional space, and training sets were formed with values of $\mathbf{x}$ for each population. The classification of unknown samples was performed by means of the Linear Discriminant Analysis (LDA) technique [22], comparing the corresponding feature vectors with both training sets. By means of this algorithm a unit vector $\mathbf{w}$ is found which maximizes the separation between classes when the data points are projected onto it. For an unclassified data point $\mathbf{x}$, the distance $d_{i}$ to the population mean $\mu_{i}$ along the direction given by $\mathbf{w}$ is

$$
(i=N, L)
$$

where the subindexes $N$ and $L$ refer to NMuMG and LM3 cells, respectively. This procedure reduces the number of dimensions to one, and the ratio between distances $R_{d}=d_{L} / d_{N}$ makes it possible to determine whether $\mathbf{x}$ most likely comes from an NMuMG $\left(R_{d}>1\right)$ or LM3 $\left(R_{d}<1\right)$ cell culture.

\section{Design and manufacture of the microelectrode array}

The complete manufacture details are given in table 1. Glass was used as substrate material due to its biocompatibility. The substrates were obtained from $25 \times 75 \mathrm{~mm}^{2}$ commercial microscope slides which were cut with a diamond cutter in three equallysized squares of $25 \times 25 \mathrm{~mm}^{2}$. The substrate was first cleaned in an ultrasonic bath with acetone, isopropyl alcohol and deionized water in order to remove any particles attached to its surface, which could impede the subsequent deposition of the metal layer, and it 
was then treated with oxygen plasma (Plasma-Preen I Plasma Cleaner) in order to remove all remaining thin layers of residuals (especially the organic compounds) [23]. Next, a metal layer was deposited on the clean substrate by means of a sputter-coater device (Pelco SC-6). Due to its biocompatibility, Au is the preferred material for the active electrodes (both the microelectrodes and the counterelectrode). However, this material shows a poor adhesion to glass, making it difficult to obtain a stable metal layer over the substrate surface. Nevertheless, this behavior is not characteristic of the material commonly known as "red gold", an $\mathrm{AuCu}$ alloy with a composition of $75 \% \mathrm{Au}$ and $25 \% \mathrm{Cu}$ in mass (atomic ratio of approximately 1:1) which adheres strongly to glass but has the disadvantage of being less biocompatible than $\mathrm{Au}$. We then performed the deposition of a $57 \mathrm{~nm}$ thick $\mathrm{AuCu}$ layer followed by the deposition of a $100 \mathrm{~nm}$ thick $\mathrm{Au}$ layer. It is worth noting that the exposed surface of the metal layer had only Au atoms (i.e. the cells are in contact only with gold during an experiment). The deposition of the metal layers was followed by another ultrasonic cleaning with acetone, isopropyl alcohol and deionized water.

The next step involved the deposition of a photoresist material ( $\mu$ Posit 1400-31, positive photoresist) through a lithography process. The resulting photoresist pattern coincided with the desired metal pattern in the MEA as shown in Figure 1 (left). As can be seen, the MEA has a total of 20 microelectrodes, which are equally distributed on four different quadrants. The counterlectrode is located in the center of the array and has the shape of a plus sign $(+)$. The dimensions of the counterelectrode were selected in such a way that $A_{c}>300 A_{a}$, where $A_{a}$ is the area of a microelectrode and $A_{c}$ is the area of the counterelectrode. Each microelectrode has its own track and pad, so that measurements can be carried out individually on each one. The non-covered parts of the metal layers were removed by means of the Reactive Ion Etching (RIE) technique (AJA International equipment) with argon (Ar) ions. The etching was carried out in a pulsated fashion, as described in Table 1, in order to avoid an increase of the sample temperature over the glass transition temperature of the photoresist. The remaining photoresist layer was then removed by means of a treatment with acetone in ultrasound followed by a short-time exposure to oxygen plasma. The resulting sample consisted of the desired metal pattern over the glass substrate.

The next step in the manufacture of the MEAs was the deposition of an isolation layer, which covered the whole surface except for the connection pads and the electrodes. The selected isolation material was the same photoresist used in the first lithography step, namely the $\mu$ Posit 1400-31, because it has an extremely low electrical conductivity and ean be easily deposited by lithography. Figure 1 (right) shows the mask used in this second lithography process. After the deposition of the isolation layer, the MEAs were treated again with oxygen plasma. This step had two purposes, namely the removal of any thin organic residual layers over the exposed metal layer and the enhancement of cellular adhesion through the increase in the hydrophilicity of the surfaces, as described in [23]. However, because of the organic nature of the photoresist material, it reacts with the oxygen plasma and thus this treatment must be carried out very carefully in order to 


\section{Microelectrode array for detection of cancer cells}

avoid the removal of the isolation layer. Finally, a cylindrical glass chamber was mounted on each MEA using silicon glue. Each well had a substrate area of approximately 104 $\mathrm{mm}^{2}$ and a total volume of $1100 \mu \mathrm{L}$. The chambers were previously deeply cleaned and sterilized with bleach.

The electrical connection between the different electrodes and the measurement devices was performed with a multi-wire planar cable soldered to a dot matrix board and attached to a polycarbonate plate, as shown in Figure 2. Spring loaded pins (P75LM) were soldered to each wire though the holes in the dot matrix board. The MEA was mounted on a polytetrafluoroethylene (PTFE) platform and the polycarbonate plate was mounted on top of it. By means of standard screws, a uniform pressure was then applied between the platform and the polycarbonate plate, allowing the spring loaded pins to effectively connect each pad with its corresponding wire. In order to enhance the electrical connections, a drop of silver conducting paint was deposited on each pad.

\subsection{Microelectrode sizes}

Commercial microelectrodes used in previous works [13] have an area of $5 \times 10^{4} \mu^{2}$, while each cell in a monolayer occupies an approximate areał of $177 \mu \mathrm{m}^{2}$ [24]. This means that the signal coming from a microelectrode of this type contains information of approximately 282 cells. When working with a cell line, the characteristics of individual cells are very similar and the signals obtained with microelectrodes of that size in different portions of the same culture are very similar as well. However, in a mixed culture these properties will/probably not be homogeneous and it is expected that the electrical responses measured with large microelectrodes, in certain portions of the culture where both types of cells are present, are different from those obtained with the corresponding cell lines. A feature vector with mixed information could be located within either population, increasing the probability of incorrect classification by the LDA algorithm $\S$. We therefore decided to carry out experiments with microelectrodes of a typical size $(211 \mu \mathrm{m})$ and with others of a smaller size (57 $\mu \mathrm{m}$, closer to cell size), in order to analyze the effect of this variable on the discrimination capacity of the method.

\subsection{Testing of the microelectrode array}

In order to test the manufactured sensors and compare different sizes and materials, impedance measurements of several naked (i.e. with culture medium but devoid of cells) microelectrodes were carried out. The results are shown in Figure 3, where equivalent resistance and capacitance spectra are plotted both for manufactured and commercial microelectrodes.

$\ddagger$ This is a registered value for MDCK cells. However, it gives an approximate idea of the order of magnitude.

$\S$ In this case, an incorrect classification is represented by a false negative, i.e. the failure to detect cancer cells in a sample. 
Table 1. Steps involved in the manufacture of the $\mathrm{AuCu} / \mathrm{Au}$ microelectrode array presented in this work. The parameter values correspond to the optimum conditions and are related to the specific devices described in the text.

As can be seen, the resistance and capacitance values of the naked microelectrodes depend strongly of their size, and the material used in the manufacture. Particularly, for $\mathrm{AuCu} / \mathrm{Au}$ microelectrodes an increase in resistance and a decrease in capacitance is observed with decreasing microelectrode diameter. This is in concordance with the naked electrode model proposed for this type of electrode/electrolyte system [25], in which the constriction resistance (high-frequency asymptotic value) is inversely proportional to the microelectrode diameter. The capacitance, on the other hand, is proportional to the microelectrode area and therefore decreases with the square of the equivalent diameter.

Regarding the material used in their manufacture, we found that the presence of $\mathrm{Cu}$ decreases the microelectrode impedance by increasing the capacitance values and decreasing the resistance values. This can be observed by comparing the spectra of the manufactured $\mathrm{AuCu} / \mathrm{Au}$ microelectrodes $(211 \pm 7.6 \mu \mathrm{m})$ with the commercial $\mathrm{Au}$ microelectrodes $(250 \mu \mathrm{m})$. The latter, although having a greater surface area, show capacitance values significantly lower than the former, and the reciprocal occurs with the resistance at low frequencies. These differences could be caused by the fact that $\mathrm{Cu}$ has a higher electrical conductivity than Au. 


\section{Microelectrode array for detection of cancer cells}

\section{Analysis of independent cultures of NMuMG and LM3 cells}

In order to determine the nature of an unknown sample by applying the technique developed in [13], it is necessary to know the discriminant vector $\mathbf{w}$, the projections of the population means corresponding to both cell types on the direction given by $\mathbf{w}$ and the projection of $\mathbf{x}$ (feature vector of the sample under study) on the same direction. The location of this last point with respect to the projections of the population means allows to classify the unknown sample as normal or cancerous, as explained in Section 2.3. In a previous work [13], the discriminant vector $\mathbf{w}$ was determined for measurements carried out with commercial Au microelectrodes measuring $250 \mu \mathrm{m}$ in diameter and under specific measurement conditions. In this work, however, a slightly different measurement protocol and other microelectrodes were used, so that a different value of $\mathbf{w}$ was expected. The first step towards the classification of an unknown sample was, therefore, the analysis of NMuMG and LM3 cells cultured individually in order to determine the value of $\mathbf{w}$ under the new measurement conditions.

The results obtained with independent cultures of NMuMG and LM3 cells for the two different microelectrode sizes are presented in Table 2. Each characteristic feature was analyzed by means of the F, t and Kolmogorov-Smirnov (KS) tests as described in [13], and probabilities $p$ were computed for each test. Accordingly, features that presented values of $p<1 \times 10^{-3}$ under the KS test and one of the other tests were considered as relevant from a discrimination point of view. As can be seen, only 7 of the 26 features presented a good discriminant capácity for the $57 \mu \mathrm{m}$ microelectrodes, while the number of relevant features was 13 for the $211 \mu \mathrm{m}$ microelectrodes, closer to the results obtained with commercial $250 \mu \mathrm{m}$ microelectrodes, where the number of relevant features was 15 [13]. Normally, a higher number of relevant features would result in a better discrimination between both populations.

Small electrodes measuring $57 \mu \mathrm{m}$ in diameter (approximately $1964 \mu \mathrm{m}^{2}$ in area) have the advantage of being covered only by a small number of cells (between 10 and 20), which would reduce the probability of obtaining mixed signals in a culture of normal and cancerous cells. However, they present complications that were analyzed in a previous work [26]. On the one hand, the impedance of these microelectrodes is greater than that of the larger ones, as shown and explained in Section 3.2. This reduces the sensitivity of the device to the presence of cells, since the cell's impedance is the same regardless of the size of the microelectrode $\|$, and this was reflected in the normalized resistance and capacitance values. This, in turn, has an effect on the quality of the features obtained during the wounding phase. More precisely, the amplitude of the wounding voltage is constant and, by Ohm's law, this results on smaller currents flowing through the cells over the smaller electrodes. In this phase, the features are obtained by modeling the

$\|$ Actually, this depends on the way in which the cells adhere to the surface of the microelectrodes. In this case, the cells are always in contact with the same material $(\mathrm{Au})$, so it is expected that they all behave in the same way. This makes reasonable the assumption that their impedance will be identical regardless of the size of the microelectrodes. 
Table 2. Comparison between the discriminant capacity of the different features for the manufactured microelectrodes. The features marked with the + sign are considered relevant in the proposed discrimination model. They were selected by means of the $\mathrm{F}$, t and Kolmogorov-Smirnov tests as described in a previous work [13]. G: growth phase. C: confluence phase. W: wound phase. H: healing phase.

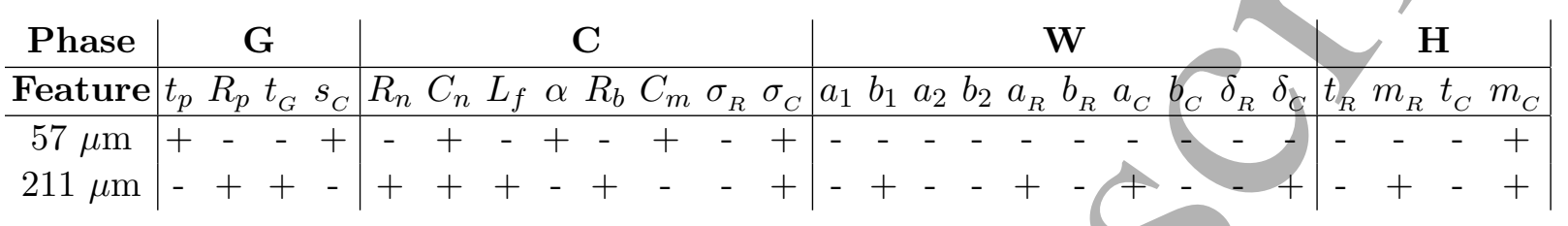

dynamics of the impedance changes during the application of the wounding signal [13], and these changes depend strongly on the nature of the cells and the amplitude of the excitation current (smaller currents induce lower rates of impedance change). This could explain the fact that the corresponding features are very similar for both cell types in the smaller electrodes, resulting in a low discriminant capacity as observed in Table 2 (none of the features obtained during the wounding phase showed a high discriminant capacity for the $57 \mu \mathrm{m}$ electrodes). On the other hand, the parameters of the model of Giaever and Keese in microelectrodes of this size had very different values from those corresponding to larger microelectrodes. This was also observed in a previous work [26] and attributed to the fact that the model does not consider the existence of currents that flow radially out of the microelectrode (effect that increases significantly as the size of the microelectrode decreases). The parameters determined with this model for small microelectrodes are then incompatible with the values calculated by other methods, and therefore lose relevance from a biological point of view. However, these characteristic parameters still provide valuable information contributing to the discrimination between NMuMG and LM3 cells, so they were not omitted in the analysis carried out in this work.

Figure 4 shows the results of a LDA analysis (refer to Section 2.3 for details) carried out for both manufactured microelectrode sizes. As can be seen, a very clear separation between populations of NMuMG and LM3 cells was obtained for $211 \mu \mathrm{m}$ electrodes, where all the classifications produced by the LDA algorithm were correct. On the other hand, the results with the $57 \mu \mathrm{m}$ microelectrodes presented $23.5 \%$ of false positives and $5.6 \%$ of false negatives. Because of the small amount of relevant features and the high rate of incorrect classifications, we concluded that the $57 \mu \mathrm{m}$ microelectrodes would not be suitable for detecting cancerous cells in a mixed culture and decided to use only the $211 \mu \mathrm{m}$ microelectrodes for that purpose.

\section{Detection of cancer cells in mixed cultures}

In this section we present the first results of measurements carried out on mixed cultures of NMuMG and LM3 cells using $\mathrm{AuCu} / \mathrm{Au}$ microelectrodes measuring $211 \mu \mathrm{m}$ in diameter. The cultures were prepared by applying the seeding protocol described in 
Section 2.1 to two cell suspensions in parallel (one of NMuMG cells and one of LM3 cells) and finally combining both of them to form a single suspension containing approximately $50 \%$ normal cells and 50\% cancerous cells with a final density of approximately $1 \times 10^{6}$ cells $/ \mathrm{ml}^{-1}$. In each experiment, measurements were carried out as indicated in Section 2.2. Feature vectors $\mathbf{x}_{j}$ corresponding to each of the samples from the same mixed culture (located on the surface of the different microelectrodes on the same MEA) were determined. With these vectors and the results of the LDA analysis of Section 4, the classification algorithm was applied to determine the nature of each sample, and the results are shown in Figure 5 for two different experiments. In the figure, a qualitative spatial map showing the position of the different microelectrodes (classified either as NMuMG, LM3 or devoid of cells) is also shown.

One of the purposes of this study is to obtain a biological indicator that could be taken into account in the process of cancer diagnosis. For this indicator to be useful, it should be easy to understand and measure. Based on the results of the classification algorithm, we then defined the transformation index $I_{t}=N_{c} / N_{T}$, where $N_{c}$ and $N_{T}$ represent the number of samples classified as cancerous and the total number of samples, respectively, obtained through the analysis of the unknown culture. Values of $I_{t}$ greater than zero would indicate the presence of transformed cells in some regions of the culture, and the closer this value is to the unit, the greater the proportion of transformed cells detected. The results obtained in two different experiments are shown in Figure 5. In the first of them (Figure 5(a)), 13 samples were classified as cancerous and 4 as normal, resulting in a transformation index equal to 0.76 . In this experiment, 3 of the microelectrodes did not show variations of their electrical impedance values with respect to the naked microelectrodes, and therefore they were not included in the analysis. In Figure 5(b) the results of another experiment are shown in which the presence of cells was detected in only 11 of the 20 microelectrodes. In this case, all the samples were classified as cancerous, and therefore $I_{t}=1$. As can be seen, the results are very different considering that the experiments were carried out exactly the same way, and they are therefore not conclusive. The discrepancy on the results could be a consequence of the absence of cells in some portions of the culture area, and a possible explanation for this behavior is that both cell types grow by forming isles separated from each other and randomly distributed throughout the entire culture surface.

\section{Conclusions}

We presented the design, fabrication and testing of a microelectrode array with spatial resolution that can be used to detect the presence of cancer cells in a mixed culture. The microelectrodes were manufactured using an $\mathrm{AuCu}$ layer between the glass substrate and the exposed Au surface, and we found that this procedure decreases the microelectrode impedance and enhances its adhesion to the substrate. We employed the manufactured microelectrode array to carry out impedance measurements on NMuMG and LM3 cells. We followed a recently developed protocol which captures the differences between both 


\section{Microelectrode array for detection of cancer cells}

cell types and makes it possible, by means of the linear discriminant analysis (LDA), to classify an unknown sample as normal or cancerous.

We analyzed the effect of the microelectrode diameter on the discriminant capacity of the method. Namely, we manufactured microelectrodes measuring $57 \mu m$ and 211 $\mu m$ in diameter. Due to its bigger area, it is more likely to find both cell types during a measurement on a $211 \mu \mathrm{m}$ electrode than on a $57 \mu \mathrm{m}$ electrode, and from this point of view the smaller electrodes would be better to discriminate cells in a mixed culture. Nevertheless, more features with discriminant capacity were found for the 211 $\mu m$ electrodes, and the linear discriminant analysis confirmed that these electrodes are more suitable for detecting cancer cells than the $57 \mu \mathrm{m}$ electrodes. The $57 \mu \mathrm{m}$ electrodes presented $23.5 \%$ of false positives and $5 \%$ of false negatives, while the $211 \mu \mathrm{m}$ electrodes didn't show incorrect classifications.

Finally, we tested the $211 \mu \mathrm{m}$ microelectrodes on mixed cultures consisting of approximately 50\% NMuMG and 50\% LM3 cells. We defined the transformation index $I_{t}$, which measures the proportion of samples on a culture whose electrical response is classified as cancerous by means of the linear discriminant analysis. Although the original cell suspensions in each experiment had the same proportions of both cell types, we did not obtain the same values of $I_{t}$. As was described in the text, we found that some of the microelectrodes in the same MEA did not detect the presence of cells at all, which would mean that the cells in the mixed culture grow forming isles which are separated from each other. In this case, more research should be carried out in order to better understand the behavior of the mixed cultures before being able to achieve quantitatively reproducible results with this method. Nevertheless, the detection of cancerous cells with the manufactured/microelectrode array was achieved in all the experiments with mixed cultures.

\section{Acknowledgments}

We want to thank Dr. Soledad Bustillo (Laboratorio de Cultivos Celulares, Universidad Nacional del Nordeste, Corrientes, Argentina) for providing us the cell lines used in this paper and Pablo Vallina for his support on the construction of the measurement platform. F.E.G. acknowledges a scholarship from Comisión Nacional de Investigaciones Científicas y Técnicas (CONICET), Argentina.

\section{References}

[1] J. Hong, K. Kandasamy, M. Marimuthu, C. Choi, and S. Kim. Electrical cell-substrate impedance sensing as a non-invasive tool for cancer cell study. Analyst, 136:237-45, 2011.

[2] G. Park, C. Choi, A. English, and T. Sparer. Electrical impedance measurements predict cellular transformation. Cell Biology International, 33:429-33, 2009.

[3] A. Rahman, C. Lo, and S. Bhansali. A detailed model for high-frequency impedance characterization of ovarian cancer epithelial cell layer using ecis. IEEE Transactions on Biomedical Engineering, 56(2):485-92, 2009. 
[4] I. Heijink, S. Brandenburg, J. Noordhoek, D. Postma, D. Slebos, and A. van Oosterhout. Characterisation of cell adhesion in airway epithelial cell types using electric cellsubstrate impedance sensing. European Respiratory Journal, 35(4):894-903, 2010.

[5] H. Pick, S. Terrettaz, O. Baud, O. Laribi, C. Brisken, and H. Vogel. Monitoring proliferative activities of hormone-like odorants in human breast cancer cells by gene transcription profiling and electrical impedance spectroscopy. Biosensors and Bioelectronics, 50:431-436, 2013.

[6] L. Arias, C. Perry, and L. Yand. Real-time electrical impedance detection of cellular activities of oral cancer cells. Biosensors and Bioelectronics, 25:2225-31, 2010.

[7] T. Nguyen, T. Yin, D. Reyes, and G. Urban. Microfluidic chip with integrated electrical cellimpedance sensing for monitoring single cancer cell migration in three-dimensional matrixes. Analytical Chemistry, 85(22):1106811076, 2013.

[8] T. Tran, C. Baek, and J. Min. Electric cell-substrate impedance sensing (ecis) with microelectrode arrays for investigation of cancer cell fibroblasts interaction. PLOS ONE, 1(4):e0153813, 2016.

[9] E. Zudaire, N. Cuesta, V. Murty, K. Woodson, L. Adams, N. Gonzalez, A. Martínez, G. Narayan, I. Kirsch, W. Franklin, F. Hirsch, M. Birrer, and F. Cuttitta. The aryl hydrocarbon receptor repressor is a putative tumor supressor gene in multiple human cancers. The Journal of Clinical Investigation, 118(2):640-50, 2008.

[10] B. Al-Husein, A. Goc, and P. Somanath. Suppression of interactions between prostate tumor cell surface integrin and endothelial ICAM1 by simvastatin inhibits micrometastasis. Journal of Cellular Physiology, 228:2139-48, 2013.

[11] H. Huang, W. Wu, and Y. Wang. ASS1 as a novel tumor suppressor gene in myxofibrosarcomas: aberrant loss via epigenetic DNA methylation confers aggressive phenotypes, negative prognostic impact, and therapeutic relevance. Clinical Cancer Research, 19:2861-72, 2013.

[12] Applied BioPhysics. Ecis cultureware disposable electrode arrays. http://www.biophysics.com/cultureware.php, 2017.

[13] F. Giana, J. Bonetto, and M. Bellotti. Assay based on electrical impedance spectroscopy to discriminate between normal and cancerous mammalian cells. Physical Review E, 97:032410, 2018.

[14] Owens R. Glandular epithelial cells from mice: a method for selective cultivation. Journal of the National Cancer Institute, 52(4):1375-8, 1974.

[15] A. Urtreger, V. Ladeda, L. Puricelli, A. Rivelli, M. Vidal, E. Delustig, and E. Joffe. Modulation of fibronectin expression and proteolytic activity associated with the invasive and metastatic phenotype in two new murine mammary tumor cell lines. International Journal of Oncology, 11(3):489-96, 1997.

[16] R. Freshney. Culture of animal cells, pages 71-159. 3 edition, 1994.

[17] Applied Biophysics. Improving experiment repeatability with cysteine. Url: http://www.biophysics.com/publications/Improving Experiment Repeatability with Cysteine.pdf.

[18] J. Wegener, C. Keese, and/. Giaever. Electric cell-substrate impedance sensing (ECIS) as a noninvasive means to monitor the kinetics of cell spreading to artificial surfaces. Experimental Cell Research, 259:158-66, 2000.

[19] I. Giaever and C. Keese. Use of electric fields to monitor the dynamical aspect of cell behavior in tissue culture. IEEE Transactions on Biomedical Engineering, BME-33(2), 1986.

[20] M. Bellotti, F. Giana, and F Bonetto. Impedance spectroscopy applied to the fast wounding dynamics of an electrical wound-healing assay in mammalian cells. Measurement Science and Technology, 26:085701, 2015.

[21] C. Keese, J. Wegener, S. Walker, and Giaever I. Electrical wound-healing assay for cells in vitro. Proceedings of the National Academy of Sciences, 101:1554-9, 2004.

[22] S. Marsland. Machine Learning: An Algorithmic Perspective, chapter 10, pages 221-226. Chapman \& Hall/CRC, 2009.

[23] E. Shun'ko and V. Belkin. Cleaning properties of atomic oxygen excited to metastable state 
Microelectrode array for detection of cancer cells $2 s^{2} 2 p^{4}\left(S_{0}\right)$. Journal of Applied Physics, 102:083304, 2007.

[24] J. Wegener, A. Janshoff, and H. Galla. Cell adhesion monitoring using a quartz crystal microbalance: Comparative analysis of different mammalian cell lines. European Biophysics Journal, 28:26-37, 1998.

[25] D. Borkholder. Cell-based biosensors using microelectrodes. PhD Thesis. Standford University. Stanford, California, USA, pages 51-64, 1998.

[26] W. Bast. Desarrollo de arreglos de electrodos micromaquinados para aplicaciones en biotecnología. Tesis de la Carrera de Doctorado en Física. Instituto Balseiro, Universidad Nacional de Cuyo/Comisión Nacional de Energía Atómica. San Carlos de Bariloche, Argentina, pages 23-48, 2014. 
(a)

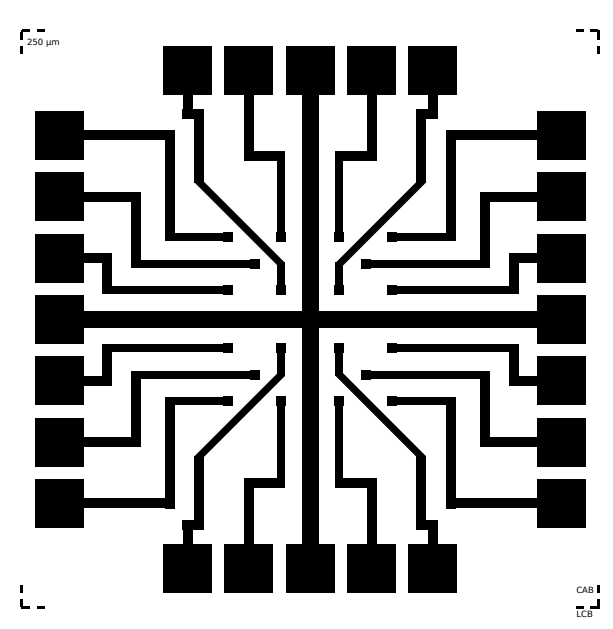

(b) Counterelectrode Microelectrode

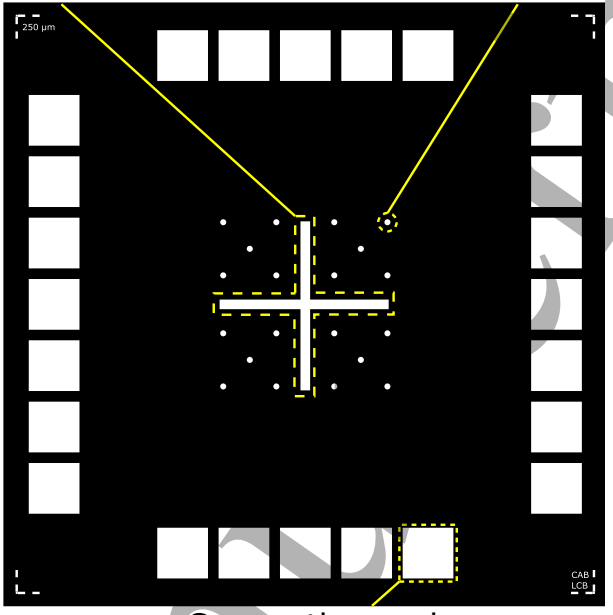

Connection pad

Figure 1. Masks used for the manufacture of the microelectrode array. Figures (a) and (b) correspond to the first and second lithography stages, respectively. The microelectrodes are equally distributed in four quadrants, each of them containing 5 microelectrodes located in the vertices and center of a square. The whole array contains a single counterelectrode located in its center, which has the shape of a plus sign $(+)$.

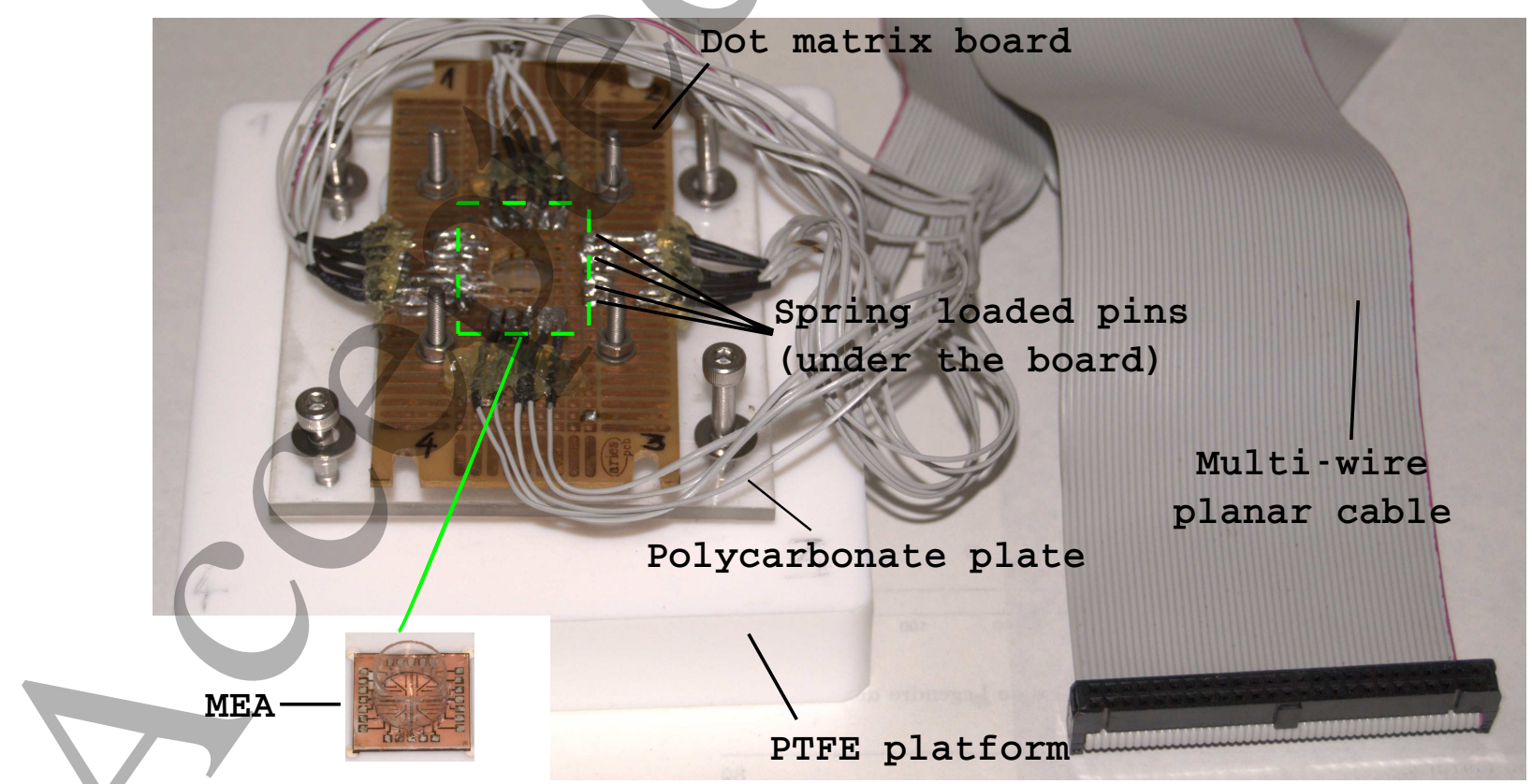

Figure 2. Photograph of the platform used to connect the microelectrode array with the measurement devices. 
Microelectrode array for detection of cancer cells

(a) $10^{5}$

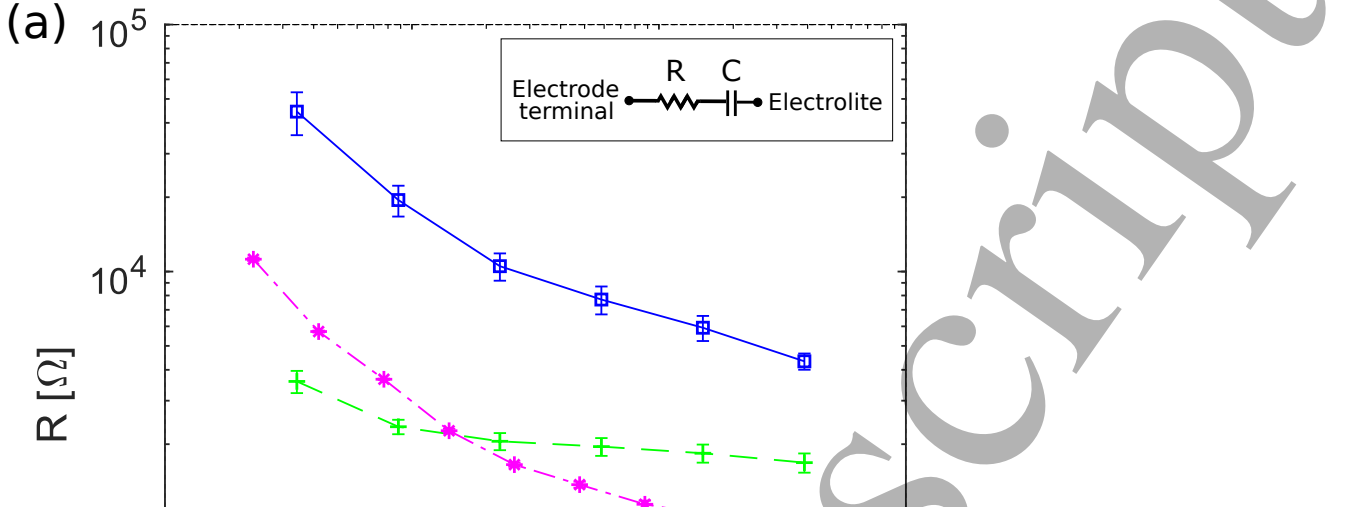

(b)

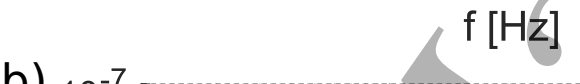

$\mathrm{f}[\mathrm{Hz}]$ $10^{5}$

Figure 3. Resistance (a) and capacitance (b) of naked microelectrodes manufactured as described in section 3 . Values for microelectrodes measuring approximately $57 \mu \mathrm{m}$ (solid blue lines) and $211 \mu \mathrm{m}$ (dotted green lines) in diameter are shown. Each point represent the average value of 20 different samples. The values corresponding to a commercial array (Applied Biophysics), which were obtained by averaging the values of 35 microelectrodes, are also shown. The error bars represent standard error 
Microelectrode array for detection of cancer cells

(a)

$$
R_{d}=1
$$

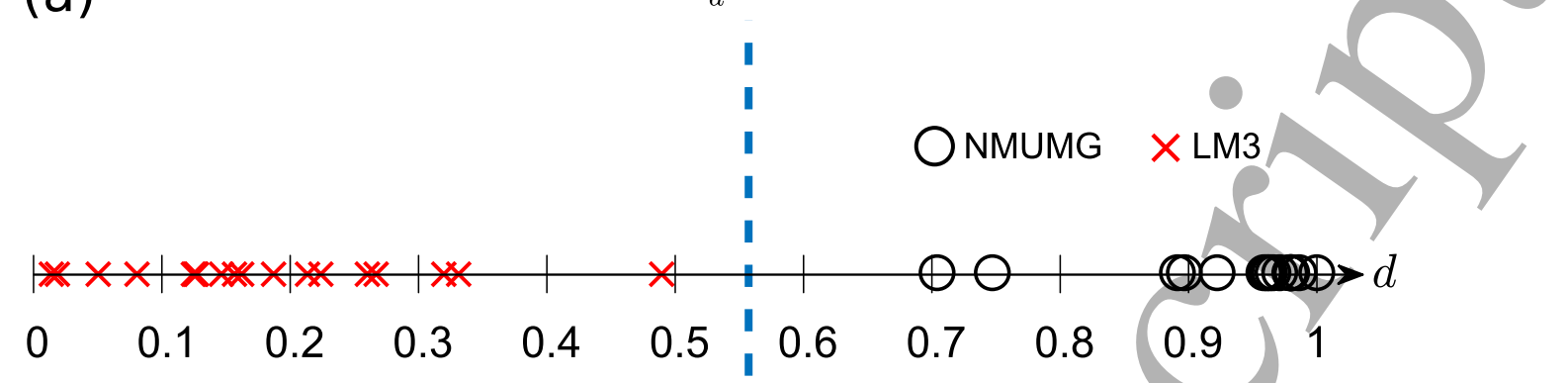

(b)

$$
R_{d}=1
$$

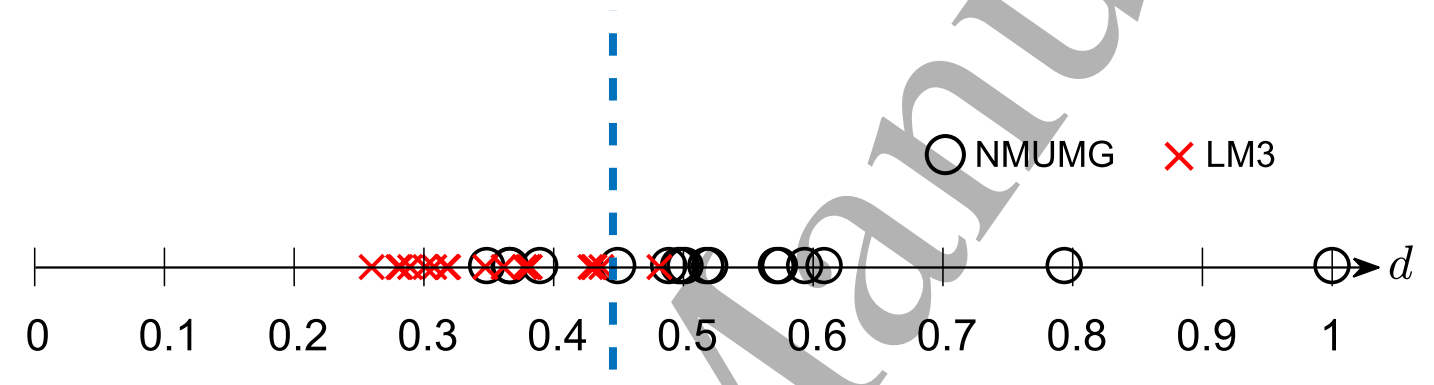

Figure 4. Euclidean distances along the direction given by the discriminant vector $\mathbf{w}$ for manufactured microelectrodes measuring $211 \mu \mathrm{m}$ (a) and $57 \mu \mathrm{m}$ (b) in diameter. Data points are represented as black circles and red crosses for NMuMG and LM3 cells, respectively. The dashed blue lines in both figures represent the boundary between both populations, where $R_{d}=1$. 
Microelectrode array for detection of cancer cells

(a) $I_{t}=0.76$

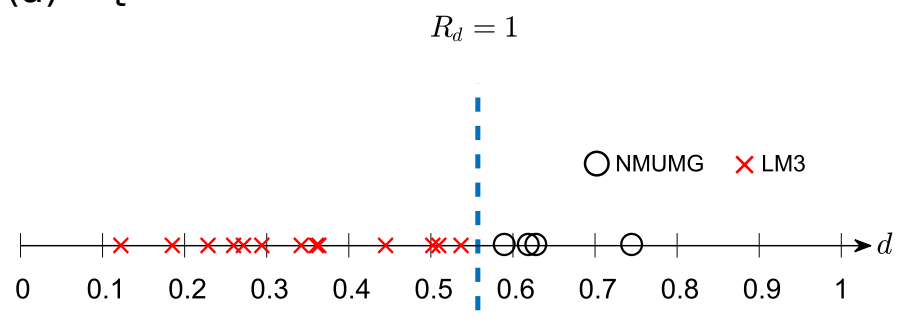

(c)

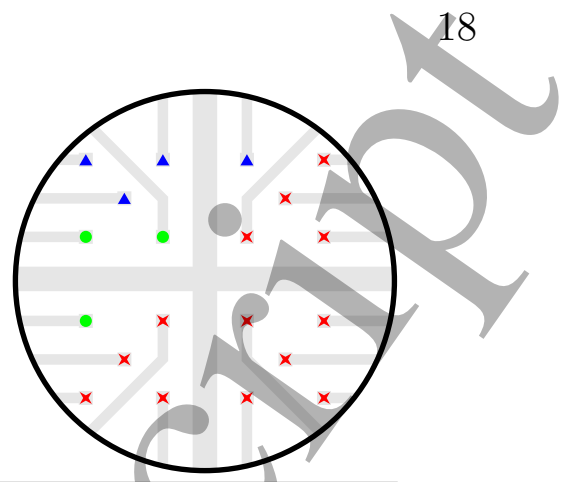

(b) $l_{t}=1.00$

(d)

$$
R_{d}=1
$$

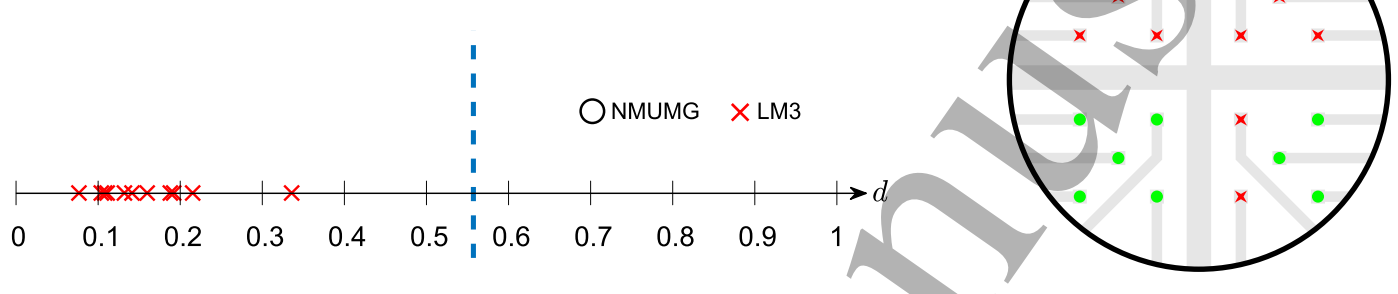

Figure 5. Classification of the different samples within a mixed culture by means of the discrimination algorithm. Figures (a) and (c) show the LDA analysis and a qualitative spatial map of the microelectrodes in a mixed culture, respectively, whereas figures (b) and (d) correspond to another repetition of the same experiment. In figures (c) and (d), the blue triangles represent microelectrodes whose electrical response was classified as NMuMG, the red crosses were classified as LM3 and the green circles represent microelectrodes whose electrical response was similar to the naked electrode's response. The transformation index $I_{t}$ is indicated in each case. 